\title{
The Tightness of the Cotton Swabs Meshing Influences the Chances of Getting Conclusive DNA Profiles
}

Mariève J Rocque ${ }^{1}$, Sarah L Leake ${ }^{1 *}$, Marie-Pierre Milon ${ }^{1}$ and Vincent Castella ${ }^{2}$

${ }^{1}$ School of Criminal Justice, Institut de police Scientifique, University of Lausanne, Batochime, 1015 Lausanne Dorigny, Switzerland

${ }^{2}$ Forensic Genetics Unit, University Center of Legal Medicine Lausanne and Geneva, Rue du Bugnon 21, CH-1011 Lausanne, Switzerland

\begin{abstract}
Objective: The chance of obtaining a conclusive DNA profile strongly depends on the quantity of biological material that can be recovered from a crime scene sample. Optimizing the collection strategy is therefore of prime interest. A difference in the level of tightness of the cotton meshed around the shaft has been observed between manufacturers and is hypothesized to affect the collection and subsequent release capacity of cotton swabs. Consequently, we compared the performance of cotton swabs from two different suppliers: Applimed SA and Dryswab ${ }^{\mathrm{TM}}$.
\end{abstract}

Methods: These swabs were used to recover $50 \mathrm{ml}$ of blood, either pure or diluted (1:1000 and 1:5000), deposited on both smooth and absorbent surfaces. Performance was compared in terms of ease of use, concentration of extracted DNA, and quality of DNA profiles. DNA quantification was obtained by real-time PCR using the Quantifiler ${ }^{\mathrm{TM}}$ Human DNA Quantification Kit. Evaluation of DNA profiles was based on profiles obtained using AmpFISTR ${ }^{\circledR}$ NGM SElect ${ }^{\mathrm{TM}}$ PCR Amplification kit.

Results: When considering smooth surfaces, recovered DNA was more concentrated when using the Dryswab ${ }^{\mathrm{TM}}$ than the Applimed SA cotton swab. More precisely, DNA concentrations ranged from 15.7 to $28.8 \mathrm{ng} / \mathrm{ml}$ and 6.7 to $21.2 \mathrm{ng} / \mathrm{ml}$, respectively for samples of pure blood. The same trend was observed for the absorbent surface, with 2.0 to $5.0 \mathrm{ng} / \mathrm{ml}$ and 0.9 to $1.4 \mathrm{ng} / \mathrm{ml}$, respectively.

Conclusion: Our results illustrate that different cotton swabs produce different results in terms of ease of use and quantity of recovered DNA and this should be taken into consideration when choosing which swab to use at both the crime scene and laboratory. More specifically, results from the present study suggest that looser meshing of the cotton fibres forming the swab is preferred.

Keywords: Forensic science; DNA typing; Cotton swab; Applimed SA swab; Dryswab ${ }^{\mathrm{TM}}$

\section{Introduction}

An increasing number of studies have shown that DNA profiles can be obtained from minute amounts of DNA, e.g. [1,2]. Evidently, this is of interest in the field of forensic genetics whereby the generally accepted methodology for collection is swabbing or cutting. With an assortment of swabs available on the market, this question is of interest to ensure that a maximum amount of DNA is collected and subsequently released from the swab. Allowing for greater quantities of DNA to be collected will result in profiles of better quality, which will be beneficial for investigative purposes and in court.

Previous studies have indicated that the capacity for collecting and releasing material may vary between collection devices [3-6]. More specifically, Hansson et al. [5] tested different collection methods, including the Dryswab ${ }^{\mathrm{TM}}$ cotton swab (Medical Wire \& Equipment, Corsham, UK) and the Scenesafe FAST ${ }^{\mathrm{TM}}$ Minitape (Scenesafe Ltd., Burnham-on-Crouch, UK). Additionally, Brownlow et al. [6] compared cotton and nylon flocked swabs for the collection of dried saliva. Nevertheless, very few studies, if any, have documented the efficiency of cotton swabs produced by different companies, for the collection and release of DNA.

In cotton swabs, the cotton is meshed around the shaft with different levels of tightness, depending on the manufacturer. It is hypothesized that a tighter mesh makes the cotton almost hydrophobic, thereby decreasing the quantity of DNA collected.

The amount of DNA transferred during contact depends on a variety of parameters; good donor versus poor donor, type and area of the surface touched, previous touches, moisture levels, etc. [1,2]. Similarly, DNA concentrations from saliva have also been shown to vary between donor and between times of day [7]. We have therefore chosen blood as the biological material in order to reduce variability between samples in terms of initial DNA concentration, which is expected to fall between $20-40 \mathrm{ng} / \mu \mathrm{L}$ as per Lee and Ladd [8]. Factors such as the visibility of blood, which allows for more accuracy in determining the area to be swabbed during collection, as well as accessibility to a sufficient number of blood samples, also played a role in the choice of biological material for this study.

The goal of this study was to evaluate the performance of two kinds of cotton swabs used for forensic purposes in Switzerland and differing in their tightness. Performance was evaluated in terms of the swabs' capacity to collect and subsequently release DNA as well as in terms of the quality of DNA profiles. The sterile medical applicator supplied by Applimed SA (Applimed SA, Châtel-Saint-Denis, Switzerland) and the Dryswab $^{\mathrm{TM}}$ sterile cotton swab supplied by Medical Wire \& Equipment

*Corresponding author: Sarah L Leake, School of Criminal Justice, Institut de police Scientifique, University of Lausanne, Batochime, 1015 Lausanne Dorigny, Switzerland, Tel: 41786757196; E-mail: sarahlouise.leake@gmail.com

Received April 10, 2014; Accepted June 02, 2014; Published June 09, 2014

Citation: Rocque MJ, Leake SL, Milon MP, Castella V (2014) The Tightness of the Cotton Swabs Meshing Influences the Chances of Getting Conclusive DNA Profiles. J Forensic Res 5: 234 doi:10.4172/2157-7145.1000234

Copyright: @ 2014 Rocque MJ, et al. This is an open-access article distributed under the terms of the Creative Commons Attribution License, which permits unrestricted use, distribution, and reproduction in any medium, provided the original author and source are credited. 
(Medical Wire \& Equipment, Corsham, UK) were selected as these are respectively used by the University Centre of Legal Medicine's Genetic Unit of Western Switzerland and by the Swiss police. The Applimed SA swab is meshed more tightly. As such, it is expected that its capacity to collect and/or release DNA is inferior to the Dryswab ${ }^{\mathrm{TM}}$. Pure or diluted blood stains deposited directly on swabs, on microscope slides and on cotton were used for this purpose.

\section{Material and Methods}

\section{Samples}

Triplicates of all samples were prepared and collected by the same individual to prevent discrepancies based on variations in individuals' collection method. The collector wore proper protective equipment (mask, gloves and lab coat) during preparation, collection and subsequent steps to prevent contamination.

Blood samples were all from the same donor. In order to simulate quantities of DNA recovered from contact traces, sterile water was added to obtain the following dilutions: 1:1000 and 1:5000.

In order to evaluate the capacity of DNA release from the cotton swabs, $50 \mu \mathrm{l}$ of pure or diluted blood from one donor was dispensed directly on both types of cotton swab. Swabs were air dried over night at ambient temperature prior to DNA extraction.

The capacity of DNA collection and subsequent release was evaluated as follows: $50 \mu \mathrm{l}$ of pure or diluted blood from one donor was dispensed on microscope slides and on white cotton fabric. Prior to trace deposition, the microscope slides were washed with bleach, then with ethanol (70\%) and were exposed to UV light for 30 minutes. The white cotton fabric was also exposed to UV light for 30 minutes before the blood was deposited. Samples were allowed to dry overnight at room temperature prior to collection.

The following day DNA was collected from the traces using the entire swab surface. The swab was humidified by depositing $50 \mu \mathrm{l}$ of sterile water prior to being rotated on its long axis whilst swabbing over the trace back and forth [9] ten times for those traces on a smooth surface and 15 times for those on an absorbent surface. One single swab per item was used.

\section{DNA extraction}

DNA was extracted from the whole swab using the QIAGEN QIAshredder/QIAamp (Qiagen AG, Hombrechtikon, Switzerland) DNA extraction procedure, allowing for better recuperation of DNA than with only QIAamp [10]. Additionally, the Microcon-30 (Millipore AG, Zug, Switzerland) was used to concentrate and desalt the samples [11] in a $25 \mu$ l volume.

\section{DNA quantification}

A volume of $2 \mu$ of the extracted DNA was quantified using Applied Biosystems 7500 Fast Real-Time PCR system with Quantifiler ${ }^{\mathrm{TM}}$ Human DNA Quantification Kit (Life Technologies Europe B.V., Zug, Switzerland) following the manufacturer's instructions. Each DNA extract was quantified in duplicate.

\section{DNA Amplification and Capillary Electrophoresis}

Volumes of $5 \mu$ of DNA extracts were amplified in triplicate with AmpFlSTR NGM SElect ${ }^{\mathrm{TM}}$ PCR Amplification kit (Life Technologies Europe B.V., Zug, Switzerland) using 30 PCR cycles on the GeneAmp PCR System 9700 (Life Technologies Europe B.V., Zug, Switzerland) and the amplicons detected using the ABI Prism 3130xl genetic analyser (Life Technologies Europe B.V., Zug, Switzerland) using standard procedures. A reporting threshold of $30 \mathrm{RFU}$ was used for reporting peaks as alleles on the electropherograms.

\section{Evaluating the quality of DNA profiles}

DNA profiles were compared with the donor's profile. For the evaluation of the quality of the profile it was assumed that the alleles that corresponded to the donor's profile were from the donor and not dropin. For the purpose of evaluating the quality of DNA profiles obtained, the percentage of correct alleles was calculated as the proportion of alleles of the donor that were effectively detected at the amelogenin locus and the 16 autosomal loci of the AmpFlSTR ${ }^{\circ}$ NGM SElect ${ }^{\mathrm{TM}}$ PCR Amplification kit (Life Technologies Europe B.V., Zug, Switzerland). The numbers of drop-out and drop-in alleles were also tabulated. Since each extract was amplified in triplicate, the average number of drop-out and of drop-in alleles of the three profiles obtained for each extract was calculated. Furthermore, the number of drop-out and drop-in alleles observed in the consensus profile (i.e. at least two occurrences out of the three profiles) was also calculated.

\section{Results and Discussion}

Methodological advances in terms of low template DNA analysis [2] have led to an increase in sensitivity in DNA analysis and consequently an increase in the number of trace DNA samples collected at crime scenes. For instance, in 2013 , about $85 \%$ of the 16,000 traces received at the Forensic genetics unit of the University Centre of Legal Medicine of Western Switzerland were epithelial cells collected on handled objects, touched surfaces or worn clothes. The collection step plays a crucial role in the quality of the profile obtained. In order to obtain high quality profiles from crime scene samples, maximum DNA must be collected and released from the collection method used. The present study hypothesized a potential difference in cotton swab performance based on the tightness of the cotton mesh around the shaft.

\section{Sample collection}

During the collection step, the Dryswab ${ }^{\mathrm{TM}}$ was much more absorbent than the Applimed SA swab. The difference in absorbency was most apparent when the cotton swabs were humidified; the Applimed SA swab required to be rotated during deposition of the blood sample and the water used to humidify the swabs prior to collection. If the swab was not rotated, the drop of blood/water would sit on top of the swab and only partially sink in. On the other hand, the same volume of blood/water deposited on the Dryswab ${ }^{\mathrm{TM}}$ would absorb much faster and without the need for as much rotation, if at all. This supports the hypothesis that tighter meshing causes the cotton to be almost hydrophobic.

Additionally, due to the looser meshing of the cotton fibres forming the bud of the Dryswab ${ }^{\mathrm{TM}}$ the fibres separated during collection. This allowed a greater surface of the cotton to come into contact with the trace and hence improve efficiency during collection.

\section{Concentrations of recovered DNA}

Firstly, we were interested in determining the capacity for DNA release of the two tested swabs. DNA extracted from samples of pure blood and 1:1000 dilutions directly deposited on the Dryswab ${ }^{\mathrm{TM}}$ showed concentrations between $28.8-53.7 \mathrm{ng} / \mu \mathrm{l}$ and $49-88 \mathrm{pg} / \mathrm{ml}$, respectively. These concentrations are within (and above) the expected values as per Lee and Ladd [8]; however, DNA concentrations for samples directly deposited on the Applimed SA were between 6.9-21.2 ng/ $\mu \mathrm{l}$ and 20-47 $\mathrm{pg} / \mu \mathrm{l}$, respectively (Figure $1 \mathrm{~A}$ ). This suggests that approximately half 
Citation: Rocque MJ, Leake SL, Milon MP, Castella V (2014) The Tightness of the Cotton Swabs Meshing Influences the Chances of Getting Conclusive DNA Profiles. J Forensic Res 5: 234 doi:10.4172/2157-7145.1000234
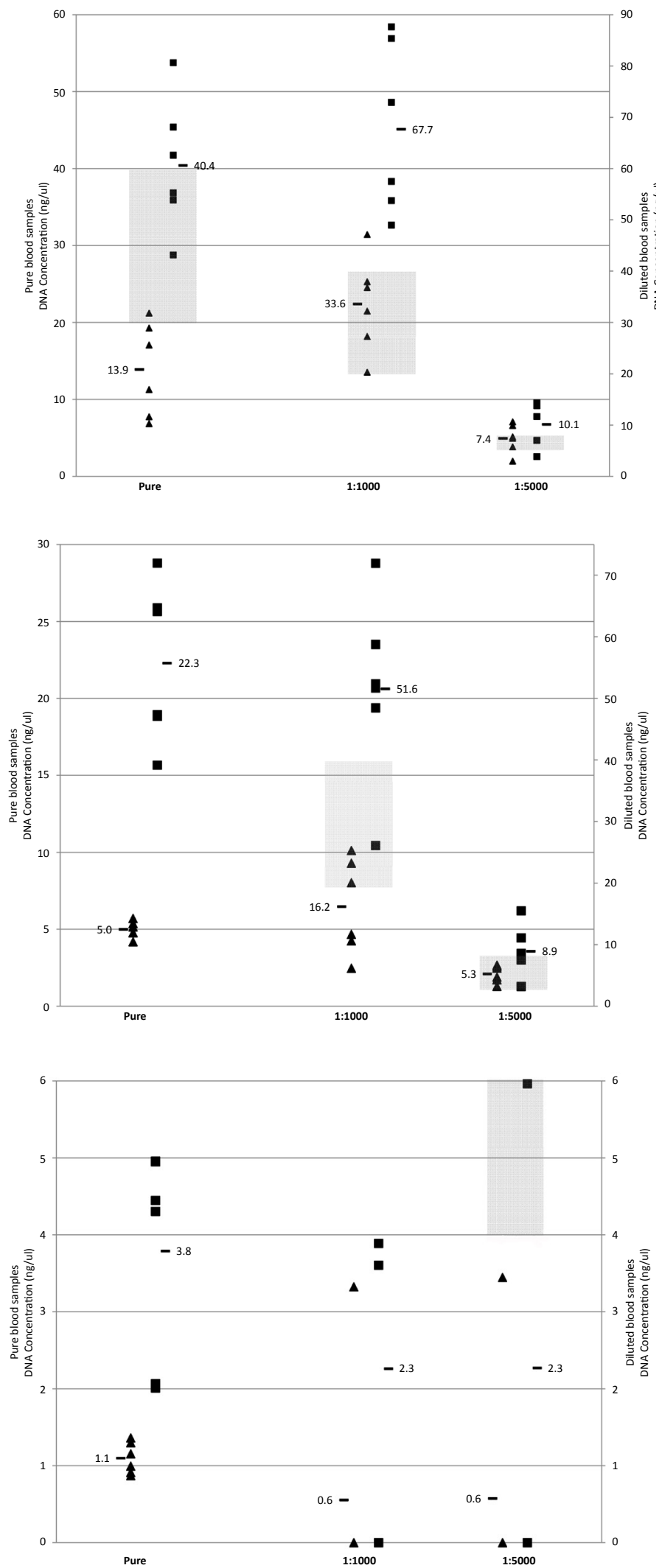

Figure 1: Comparison of DNA concentration obtained from: (A) blood directly deposited on the cotton swabs; (B) blood deposited, dried and collected from a smooth surface (microscope slide); (C) blood deposited, dried and collected from an absorbent surface (white cotton). Values of zero indicate samples that were not quantified successfully and the shaded areas represent the expected DNA concentration [12]. 
the DNA material was trapped within the Applimed SA swab, based on the values of Lee and Ladd [8]. These data therefore support a better capacity for DNA release from the Dryswab ${ }^{\mathrm{TM}}$ than the Applimed SA swab. The Quantifiler ${ }^{\mathrm{TM}} \mathrm{DNA}$ quantification kit allows for reproducible results for samples with a DNA concentration between 100 and 5000 $\mathrm{pg} / \mu \mathrm{l}$ [12]. This probably explains, at least partly, the relatively large dispersion of the results obtained for the most concentrated samples.

As the samples become less concentrated (1:5000), the range of DNA concentration overlaps between swab types and the difference between the two swabs' capacity for DNA release becomes minimal. More specifically, the Dryswab ${ }^{\mathrm{TM}}$ released between $4-14 \mathrm{pg} / \mu \mathrm{l}$ of DNA whereas the Applimed SA released between 3-11 pg/ $\mu$ l of DNA (Figure 1A). Note that these concentrations are below the detection threshold of 23 $\mathrm{pg} / \mathrm{ml}$ for the quantification method used and should be considered with extreme caution [13].

Secondly, we were interested in determining the capacity for DNA collection on smooth surface and subsequent release of the two tested swabs. Generally, a higher concentration of DNA was obtained with the Dryswab ${ }^{\mathrm{TM}}$ than with the Applimed SA swab (Figure 1B) which follows the trend observed regarding capacity for DNA release. This difference is more acute for samples which are more concentrated (pure blood and 1:1000). More specifically, the concentration ranges of DNA for samples of pure blood were 15.7-28.8 $\mathrm{ng} / \mu \mathrm{l}$ and 4.2-5.7 $\mathrm{ng} / \mu \mathrm{l}$ for samples collected from a smooth surface using the Dryswab ${ }^{\mathrm{TM}}$ and for the Applimed SA, respectively. Samples of blood diluted to 1:1000 and collected from a smooth surface allowed the extraction of $26-72 \mathrm{pg} / \mu \mathrm{l}$ of DNA for the Dryswab ${ }^{\mathrm{TM}}$ and 6-25 pg/ $\mu$ of DNA for the Applimed SA swab (Figure 1B). As the initial DNA concentration decreases (1:5000) the quantity of DNA extracted begins to overlap between the two cotton swabs tested; however, the Dryswab ${ }^{\mathrm{TM}}$ continues to generate more concentrated samples: these values range between 3-15 pg/ $\mu$ and 3-7 pg/ $\mu$, according to the type of swab. Again, these values are close to the detection threshold of the quantification method and should be considered with caution.

Thirdly, we were interested in determining the capacity for DNA collection on absorbent surface and subsequent release of the two tested swabs. In terms of samples of pure blood collected from an absorbent surface, concentrations between 2.0-4.9 $\mathrm{ng} / \mu \mathrm{l}$ and $0.9-1.4 \mathrm{ng} / \mu \mathrm{l}$ were obtained, respectively for the Dryswab ${ }^{\mathrm{TM}}$ and the Applimed SA swabs (Figure 1C). The difference in quantified DNA between smooth and absorbent surfaces is quite large (see values above) when considering pure blood samples. This can be attributed to blood being trapped in the meshes of the cotton fabric used for the absorbent surface. This underlines that when possible the cutting method should be used for traces on absorbent surfaces [14]. Moreover, the thinness of the absorbent surface resulted in further loss of sample as blood seeped through both layers of the cotton.

As for diluted samples used to simulate contact traces, DNA quantities obtained following collection from an absorbent surface were all below $8 \mathrm{pg} / \mu \mathrm{l}$ (Figure $1 \mathrm{C}$ ), and therefore, with a few exceptions, below the expected concentrations [8]. This is true regardless of the swab used and the dilution factor. Generally, the highest concentrations were obtained when the Dryswab ${ }^{\mathrm{TM}}$ was used. Collection from an absorbent surface with the Applimed SA swab resulted in the highest number of unsuccessful quantifications.

Results of the three experimental design presented above suggest that the tightness of the cotton mesh of the bud has an effect, not only in its absorbency, as observed during collection, but also on its capacity to release DNA during extraction by QIAshredder/QIAamp; a tighter cotton mesh will result in an inferior capacity for DNA release. It is hypothesized that just as it is difficult for matter to absorb into the bud through the tight meshes of cotton, once it has absorbed, it is difficult for this matter to disperse out of these tight meshes. Hence, the Dryswab ${ }^{\mathrm{TM}}$ demonstrates a better capacity for release than the Applimed SA swab due to a looser meshing of the cotton forming its bud. The comparison of the quantitative data obtained for the two swabs suggests that with the Applimed SA swab about half of the DNA from the original blood stains was left on the substrate at the time of collection and another half of this DNA was further lost within the swab when the stain was resuspended.

\section{Quality of DNA profiles}

Samples collected from pure blood stains were not amplified in this study as it was expected that high quality profiles would be obtained, regardless of the collection method. Nevertheless, quantification data and DNA profile quality can be poorly correlated, particularly with low level DNA samples [13].

The quality of DNA profiles obtained from diluted samples was evaluated in terms of average number of drop-out and drop-in alleles as well as total number of drop-out and drop-in alleles present in the consensus profile (Table 1). Since the donor is homozygous at two loci, this means the total number of (correct) alleles is 32 (including the amelogenin locus).

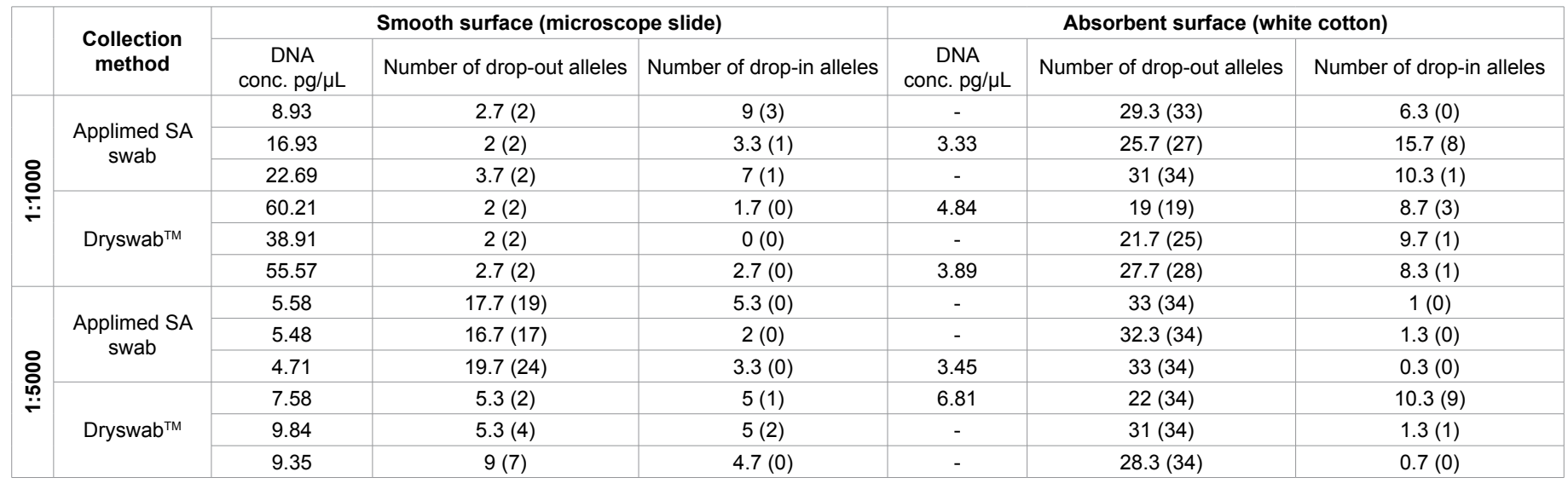

Table 1: Evaluation of the quality of DNA profiles obtained from samples of diluted blood deposited on smooth and absorbent surfaces, and collected by two different cotton swabs. An average number of drop-out and drop-in alleles are given for the three profiles obtained per extract. The numbers in brackets represent the number of drop-out and drop-in alleles still present in the consensus profile. Note that extracts obtained from pure blood samples were not amplified. 
Citation: Rocque MJ, Leake SL, Milon MP, Castella V (2014) The Tightness of the Cotton Swabs Meshing Influences the Chances of Getting Conclusive DNA Profiles. J Forensic Res 5: 234 doi:10.4172/2157-7145.1000234

Page 5 of 5

Table 1 shows that samples collected from smooth surfaces allowed for better quality profiles to be generated when compared to those collected from absorbent surfaces, i.e. fewer allelic drop-outs and dropins. Similarly, a decrease in quality of profiles is also observed as the dilution factor increases. This is concordant with observations made in relation to the quantity of DNA extracted from these respective sets of samples.

In spite of the difference in the quantity of extracted DNA from samples deposited on smooth surfaces, only a small difference in the quality of profiles between the two swabs is observed for samples that are the least diluted (1:1000). The difference in quality of these profiles is attributed to a higher occurrence of drop-in for samples collected by the Applimed SA swab (Table 1).

An obvious difference in profile quality obtained from the two cotton swabs tested during this study is observed when collecting traces from an absorbent surface; in fact, drop-out alleles are far more frequent in profiles generated from samples collected by the Applimed SA swab than by the Dryswab ${ }^{\mathrm{TM}}$. The extent of this difference is much greater than the difference in DNA concentration.

\section{Conclusions}

In order to establish whether the mesh tightness has an effect on a cotton swab's capacity to collect and subsequently release DNA, the performance of the Applimed SA cotton swab and the Dryswab ${ }^{\mathrm{TM}}$ cotton swab are compared. Performance is evaluated in terms of concentration of extracted DNA as quantified by real-time PCR using Quantifiler ${ }^{\mathrm{TM}}$ Human DNA Quantification kit and in terms of quality of DNA profiles generated using AmpFlSTR ${ }^{\circ}$ NGM SElect ${ }^{\mathrm{TM}}$ PCR Amplification kit.

The capacity of DNA release is tested by adding blood samples directly to the swabs and allowed to dry prior to extraction. The DNA quantification results of these extracts show that the Dryswab ${ }^{\mathrm{TM}}$ allows for better release than the Applimed SA swab. This is hypothesized to be due to the looser meshing of the cotton fibres in the Dryswab ${ }^{\mathrm{TM}}$; the looser meshing allows a greater contact surface between the swab and the DNA stain as the fibres separate during collection. Further, tighter meshing is hypothesized to trap DNA material during extraction.

In comparing the quantity of DNA extracted from samples collected from both smooth and absorbent surfaces, extracts from the Dryswab $^{\mathrm{TM}}$ demonstrate higher DNA concentrations.

The quality of DNA profiles is assessed in terms of percentage of correct alleles, number of drop out alleles and number of drop in alleles. Results demonstrate that the Dryswab ${ }^{\mathrm{TM}}$ out performs the Applimed SA swab with regards to the quality of DNA profiles, this is particularly true with regards to samples collected from the absorbent surface.

It can therefore be concluded that the tightness of cotton swab meshing has an influence on the quantity of DNA obtained and the subsequently generated DNA profiles. Consequently, the results of this study demonstrate the importance of testing, not only different types of collection methods made of different materials (e.g. minitape or nylon flocked swab versus cotton swab $[5,6])$, but also different brands of the same type of collection method. This is important as the manufacturing process might vary between swabs of the same type. In the case of cotton swabs, the present study demonstrates that looser meshing of the cotton is preferred.

Lastly, several studies including this one have demonstrated that the collection is variable and has an effect on the amount of DNA recovered. This demonstrates the importance of collaboration between laboratories and those responsible for trace DNA collection in order to allow for an optimized collection methodology, thus allowing for greater concentrations of recovered DNA and DNA profiles with higher power of discrimination. This collaboration is crucial because, generally, sample collection at crime scenes and the subsequent DNA analysis are not carried out by the same personnel.

\section{Acknowledgement}

The authors would like to thank Mrs Nathalie Hicks Champod for her useful comments on the manuscript.

\section{References}

1. Lowe A, Murray C, Whitaker J, Tully G, Gill P(2002) The propensity of individuals to deposit DNA and secondary transfer of low level DNA from individuals to inert surfaces. Forensic Sci Int 129: 25-34.

2. Van Oorschoot RAH, Phelan DG, Furlong S, Scarfo GM, Holding NL, Cummins MJ (2003) Are you collecting all the available DNA from touched objects? Int Congr Ser 1239: 803-807.

3. Daley P, Castriciano S, Chernesk M, Smieja M (2006) Comparison of flocked and rayon swab for collection of respiratory epithelial cells from uninfected volunteers and symptomatic patients. J Clin Microbiol 44: 2265-2267.

4. Dalmaso G, Bini M, Paroni R, Ferrari M (2008) Qualification of high-recovery flocked swabs as compared to traditional rayon swabs for microbiological environmental monitoring of surfaces. PDA J Pharm Sci and Tech 62: 191-199.

5. Hansson O, Finnebraaten M, Knutsen Heitmann I, Ramse M, Bouzga M (2009) Trace DNA collection-Performance of minitape and three different swabs. Forensic Sci Int:Gen Suppl 2: 189-190.

6. Brownlow RJ, Dagnall KE, Ames CE (2012) A comparison of DNA collection and retrieval from two swab types (Cotton and Nylon Flocked Swab) when Processed Using Three QIAGEN extraction methods. J Forensic Sci 57: 713717.

7. Kenna J, Smyth M, McKenna L, Dockery C, McDermott S (2011) The Recovery and Persistence of Salivary DNA on Human Skin. J Forensic Sci 56: 170-175.

8. Lee HC, Ladd C (2001) Preservation and Collection of Biological Evidence. Croat Med J 42: 225-228.

9. Van Oorschot RAH, Ballantyne KN, Mitchel RJ (2010) Forensic Trace DNA: A review. Investig Genet 1-14 doi: 10.1186/2041-2223-1-14.

10. Castella V, Dimo-Simonin N, Brandt-Casadevall C, Mangin P (2006) Forensic evaluation of the QIAshredder/QIAamp DNA extraction procedure. Forensic Sci Int 156: 70-73.

11. Millipore Corporation (2005) MICROCON® Centrifugal Filter Devices-User Guide.

12. John LM, Thakor A, loannou P, Kerai J, Thomson JA (2006) Validation of Quantifiler ${ }^{\mathrm{TM}}$ Human Quantification Kit for forensic casework. Int Cong 1288 762-764.

13. Cupples CM, Champagne JR, Lewis KE, Cruz TD (2009) STR Profiles from DNA samples with "undetected" or low quantifiler (TM) results. J Forensic Sci 54: 103-107.

14. Castella V, Mangin P (2008) DNA profiling success and relevance of 1739 contact stains from caseworks. Forensic Sci Int:Gen Suppl 1: 405-407. 